\section{(2) OPEN ACCESS}

\title{
Hypomethylation of a centromeric block of ICR1 is sufficient to cause Silver-Russell syndrome
}

\author{
Ken Higashimoto, ${ }^{1}$ Hijiri Watanabe, ${ }^{2}$ Yuka Tanoue, ${ }^{1}$ Hidefumi Tonoki, ${ }^{3}$ \\ Tomoharu Tokutomi, ${ }^{4}$ Satoshi Hara, ${ }^{1}$ Hitomi Yatsuki, ${ }^{1}$ Hidenobu Soejima (D) ${ }^{1}$
}

\begin{abstract}
- Additional material is published online only. To view please visit the journal online (http://dx.doi.org/10.1136/ jmedgenet-2020-106907)
\end{abstract}

${ }^{1}$ Division of Molecular Genetics \& Epigenetics, Department of Biomolecular Sciences, Saga University Faculty of Medicine, Saga, Japan

${ }^{2}$ Department of Pediatrics, Amakusa Medical Center, Amakusa, Japan ${ }^{3}$ Medical Genetics Center, Department of Pediatrics, Tenshi Hospital, Sapporo, Japan ${ }^{4}$ Department of Clinical Genetics, School of Medicine, Iwate Medical University, Morioka, Japan

\section{Correspondence to}

Dr Hidenobu Soejima and Dr Ken Higashimoto, Division of Molecular Genetics \& Epigenetics, Department of Biomolecular Sciences, Saga University Faculty of Medicine, Saga 849-8501, Japan; soejimah@cc.saga-u.ac.jp, higashim@cc.saga-u.ac.jp

Received 17 February 2020 Revised 8 April 2020 Accepted 20 April 2020 Published Online First 23 May 2020

\section{Check for updates}

(c) Author(s) (or their employer(s)) 2021. Re-use permitted under CC BY-NC. No commercial re-use. See rights and permissions. Published by BMJ.

To cite: Higashimoto $\mathrm{K}$,

Watanabe $\mathrm{H}$, Tanoue $\mathrm{Y}$, et al. $J$ Med Genet

2021:58:422-425.

\section{ABSTRACT}

Silver-Russell syndrome (SRS) is a representative imprinting disorder. A major cause is the loss of methylation (LOM) of imprinting control region 1 (ICR 1) within the IGF2/H19 domain. ICR1 is a gametic differentially methylated region (DMR) consisting of two repeat blocks, with each block including three CTCF target sites (CTSs). ICR1-LOM on the paternal allele allows CTCF to bind to CTSs, resulting in IGF2 repression on the paternal allele and biallelic expression of H19. We analysed 10 differentially methylated sites (DMSs) (ie, seven CTSs and three somatic DMRs within the IGF2/H19 domain, including two IGF2-DMRs and the H19-promoter) in five SRS patients with ICR1-LOM. Four patients showed consistent hypomethylation at all DMSs; however, one exhibited a peculiar LOM pattern, showing LOM at the centromeric region of the IGF2/H19 domain but normal methylation at the telomeric region. This raised important points: there may be a separate regulation of DNA methylation for the two repeat blocks within ICR 1; there is independent control of somatic DMRs under each repeat block; sufficient IGF2 repression to cause SRS phenotypes occurs by LOM only in the centromeric block; and the need for simultaneous methylation analysis of several DMSs in both blocks for a correct molecular diagnosis.

\section{INTRODUCTION}

Silver-Russell syndrome (SRS; OMIM 180860) is an imprinting disorder characterised by prenatal and postnatal growth restriction, relative macrocephaly, a protruding forehead, asymmetry, and feeding difficulties. ${ }^{1}$ Loss of methylation (LOM) of imprinting control region 1 (ICR1) within the IGF2/H19 domain at $11 \mathrm{p} 15.5$ is a major cause of SRS and is found in 30\%-60\% of patients. Other causative alterations are maternal UPD of chromosomes 7, 14, 16 and 20; maternal 11p15 duplication; aberrant methylation of $14 \mathrm{q} 32.2$; paternal IGF2 loss-of-function mutations; and maternal CDKN1C gain-of-function mutations. ${ }^{1}$ ICR1 is a differentially methylated region (DMR) with methylation of the paternal allele and an unmethylated maternal allele. Differential methylation of ICR1 is established during gametogenesis (ie, it is a gametic DMR), and is continuously maintained in somatic cells. ${ }^{23}$ ICR 1 is located $2 \mathrm{~kb}$ upstream of the $H 19$ gene and controls paternal expression of IGF2 (a growth factor) and maternal expression of H19 (a growth inhibitor). On the maternal allele, unmethylated ICR1 bound by CTCF functions as a chromatin insulator that prevents the interaction of the IGF2 promoter and the enhancer downstream of $H 19$, resulting in the silencing of IGF2 and the activation of H19. On the paternal allele, prevention of CTCF binding to methylated ICR1 allows the interaction between the IGF2 promoter and the enhancer downstream of $H 19$, resulting in the activation of IGF2 and the silencing of H19. ${ }^{2}$ Therefore, ICR1-LOM on the paternal allele induces repression of IGF2 expression and biallelic expression of H19 (causing an overexpression of H19), leading to the SRS phenotype. ${ }^{1}$

Human ICR1 is arranged in two repeat blocks, namely a centromeric block and a telomeric block (figure 1A). Each block contains one repetitive sequence $\mathrm{A}$ and three or four repetitive sequences B. There are seven CTCF target sites (CTSs). Among them, six CTSs are present in each repetitive sequence B (except B4) and CTS7, which is located outside of the repeat blocks. ${ }^{1}$ In addition to ICR1, the IGF2/H19 domain contains three somatic DMRs: IGF2-DMR0, IGF2-DMR2 and the H19promoter (figure 1A). These are methylated on the paternal allele and are established after fertilisation under the control of ICR $1 .^{4}$ The proper allelespecific methylation at ICR1 appears to be mediated by several DNA binding trans-factors, such as CTCF, OCT4, SOX2, ZFP57, ZBTB33 (KAISO) and YY1. ${ }^{5-7}$ CTCF, OCT4 and SOX2 are required to maintain the unmethylated status of maternal ICR $1 .^{8}$ Microdeletions (1.4-2.2 kb) abolishing one to three CTSs, single-nucleotide variants, or a small deletion of the OCT4/SOX2 target site can result in ICR1 hypermethylation. Hypermethylation causes Beckwith-Wiedemann syndrome, an overgrowth syndrome molecularly and clinically opposite to SRS. ${ }^{7-11}$ In contrast, ZFP57 is required to maintain the methylated status of many ICRs in mice, though the methylation status of ICR1 was normal in Z fp57 homozygous mutant embryos. ${ }^{12}{ }^{13}$ However, deletions including ZFP57 binding sites in SRS patients with ICR1-LOM suggest that ZFP57 is required to maintain the methylated status of paternal ICR1. ${ }^{67}$ ZBTB33 is also important for the maintenance of the methylated status of paternal ICR $1 .{ }^{5}$ However, it has not been clearly shown whether ZFP57 and ZBTB33 are involved in the aetiology of SRS.

In total, there are 10 differentially methylated sites (DMSs) within the IGF2/H19 domain, including two IGF2-DMRs, seven CTSs and the H19-promoter (figure 1A). To date, the DNA methylation status of the DMSs, excluding CTS5 
A

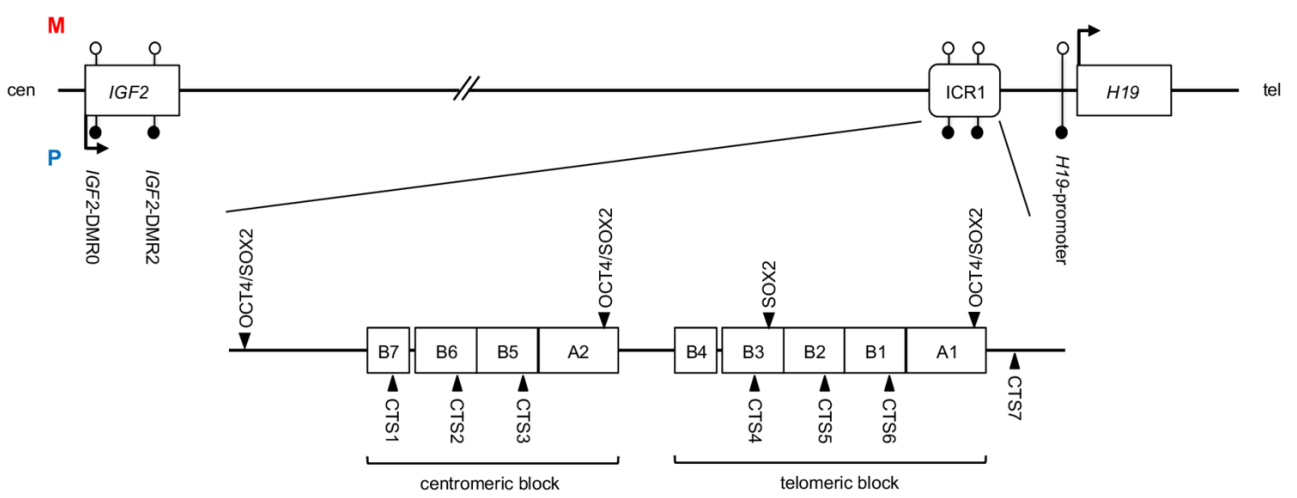

B

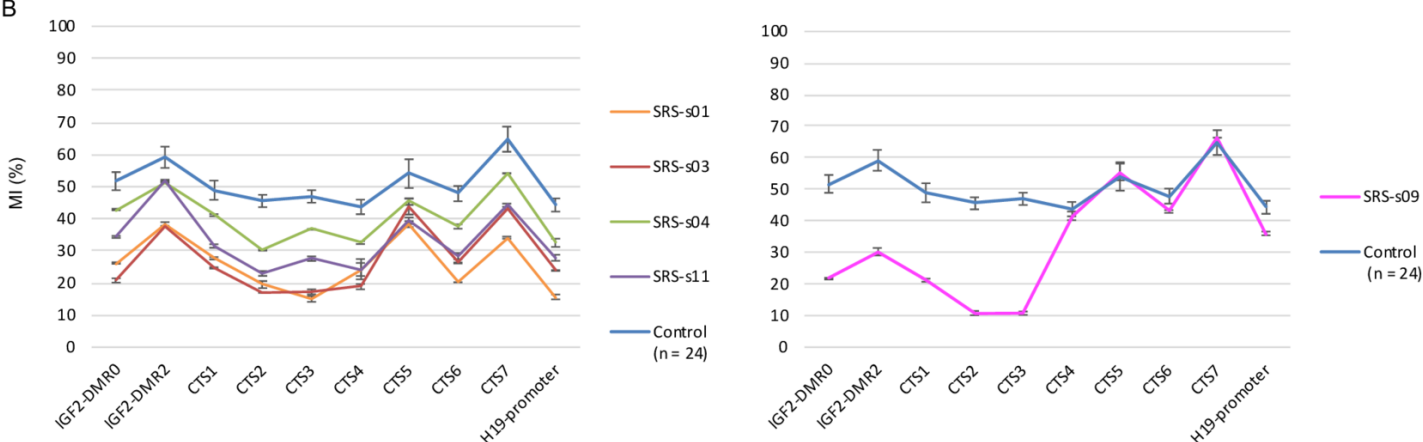

Figure 1 Structure of the IGF2/H19 domain and methylation status of patients with SRS. (A) Schematic representation of the 10 DMSs within the /GF2/ H19 domain (not to scale). The lower panel shows an enlarged view of ICR1. ICR1 is arranged in two repeat blocks, one centromeric and one telomeric. Each block contains one repetitive sequence $A$ and three or four repetitive sequences B. The seven CTSs, three OCT4/SOX2 motifs and one putative SOX2 consensus motif are shown by arrowheads. The transcription directions of the genes are indicated by arrows. (B) Methylation status of 10 DMSs in patients with SRS and control subjects. Left: average MIs with SD of two independent bisulfite-pyrosequencing data at each DMS from four patients with SRS. Average MIs with SD of controls. Right: average MIs with SD of SRS-s09 and control subjects. SRS-s09 showed a peculiar pattern of LOM, exhibiting LOM at DMSs within the centromeric region of the IGF2/H19 domain and normal methylation at DMSs within the telomeric region. The bisulfite-pyrosequencing assessment of patients was performed in duplicate. The Mls of all patients and control subjects are shown in online supplementary tables S1 and S3, respectively. cen, centromere; CTSS, CTCF target sites; DMR, differentially methylated region; DMSs, differentially methylated sites; ICR1, imprinting control region 1; LOM, loss of methylation; MI, methylation index; $\mathrm{M}$, maternal allele; P, paternal allele; SRS, Silver-Russell syndrome; tel, telomere; open lollipop, unmethylated DMS; black lollipop, methylated DMS.

and IGF2-DMR2, has been investigated in SRS patients with ICR1-LOM. Uneven methylation profiles among the DMSs and normal methylation at CTS1 and CTS7 have been reported. ${ }^{14}$ In this study, we examined all 10 DMSs in five SRS patients with ICR1-LOM. We found even levels of hypomethylation in four patients. However, one patient exhibited an unusual pattern of LOM, showing LOM at the centromeric region of the IGF2/H19 domain and normal methylation at the telomeric region. This peculiar LOM raised important points in terms of imprinting mechanisms and molecular diagnoses.

\section{MATERIALS AND METHODS}

Patients with SRS and healthy controls

Five patients with SRS were analysed in this study. The NetchineHarbison clinical scoring system was used as the clinical

Table 1 The Netchine-Harbison clinical scores of patients with SRS in this study

\begin{tabular}{|c|c|c|c|c|c|}
\hline Clinical criteria & SRS-s01 & SRS-s03 & SRS-s04 & SRS-s09 & SRS-s11 \\
\hline SGA (birth weight and/or birth length) & $\checkmark$ & $\checkmark$ & $\checkmark$ & $\checkmark$ & $\checkmark$ \\
\hline Postnatal growth failure & & $\checkmark$ & $\checkmark$ & $\checkmark$ & $\checkmark$ \\
\hline Relative macrocephaly at birth & $\checkmark$ & & $\checkmark$ & $\checkmark$ & $\checkmark$ \\
\hline Protruding forehead & $\checkmark$ & $\checkmark$ & $\checkmark$ & & $\checkmark$ \\
\hline Body asymmetry & $\checkmark$ & $\checkmark$ & & & $\checkmark$ \\
\hline Feeding difficulties and/or low BMI & & $\checkmark$ & $\checkmark$ & $\checkmark$ & \\
\hline Age & 2 years & 3 years & 10 years & 2 years & $\begin{array}{l}1 \text { year } \\
6 \text { months }\end{array}$ \\
\hline Sex & Female & Male & Male & Male & Female \\
\hline
\end{tabular}

A clinical diagnosis is considered if a patient has at least four of the six criteria.

BMI, body mass index; SGA, small for gestational age; SRS, Silver-Russell syndrome. 
diagnostic criterion for SRS. All patients scored positive for at least four of the six components of the criteria and were diagnosed with clinical SRS (table 1). ${ }^{1}$ LOM at CTS3 within ICR1 was confirmed in all patients (online supplementary figure S1A). Normal methylation (approximately 50\% of methylation index (MI)) of ICR2 due to maternal methylation and paternal unmethylation was also confirmed in all patients (online supplementary figure S1B). Maternal duplication of $11 \mathrm{p} 15$ and maternal UPD of chromosome 7 were excluded (online supplementary figure S2 for SRS-09; data not shown for other patients). These indicated that isolated ICR1-LOM was a causative alteration in these patients. In addition, healthy children $(\mathrm{n}=24 ; 12$ girls and 12 boys; mean age: $3.8 \pm 2.4$ years), were used as control subjects (online supplementary table S1). Genomic DNA was extracted from peripheral blood leucocytes of all subjects using the FlexiGene DNA Kit (Qiagen, Hilden, Germany).

Written informed consent was provided by all parents/ guardians.

\section{DNA methylation analysis}

We investigated the methylation status of 10 DMSs within the IGF2/H19 domain (ie, CTS1-7, IGF2-DMR0, IGF2-DMR2 and the H19-promoter) using bisulfite-pyrosequencing, as previously described (figure $1 \mathrm{~A}) .{ }^{11}$ The methylation status of $M E G 3$-DMR was also investigated through bisulfite-pyrosequencing. ${ }^{15}$ MIs were calculated as the average methylation of $\mathrm{CpG}$ sites within each DMS. The bisulfite-pyrosequencing assessment was performed in duplicate. Bisulfite sequencing of CTS5 was also performed as follows: the bisulfite PCR products were cloned into a pT7Blue T-Vector (Merck KGaA, Darmstadt, Germany) and individual clones were sequenced. Primers for CTS5 bisulfite sequencing are shown in online supplementary table S2.

\section{Sequencing analysis}

Microdeletions/insertions in ICR1 were screened for using longrange PCR encompassing the entire ICR1 region (GRCh37/ hg19, chr11: 2017 992-2 025 550), including seven CTSs, two OCT4/SOX2 sites and 12 ZFP57 sites. Sanger sequencing was performed to investigate the variants in the entire ICR1 region, as previously described. ${ }^{10}{ }^{11}$ The coding regions of CDKN1C were also sequenced, as previously described. ${ }^{16}$ Furthermore, the coding regions of IGF2 and ZFP57 were sequenced. Primers for sequencing analyses of IGF2 and ZFP57 are shown in online supplementary table S2.

\section{Analyses of short tandem repeats}

Short tandem repeat markers (STR) on chromosomes 7, 11, 14, 16 and 20 were analysed to investigate maternal UPD. Primers for STR analyses are shown in online supplementary table S2.

\section{Copy number analysis of the IGF2/H19 domain}

Copy number analysis of the IGF2/H19 domain was performed by multiplex ligation-dependent probe amplification (MLPA) with a ME030-C3 BWS/RSS kit according to the protocol provided by the manufacturer (MRC-Holland, Amsterdam, The Netherlands).

\section{RESULTS}

We analysed the methylation status of all 10 DMSs within the IGF2/H19 domain using bisulfite-pyrosequencing to determine whether uneven methylation profiles of DMSs were present in our patients. The MIs of all DMSs in four patients (ie, SRS-s01, SRS-s03, SRS-s04, SRS-s11) were lower than those observed in the healthy controls (figure 1B left, online supplementary table S3). $\Delta$ Methylation $(\Delta \mathrm{me})$ of the patients, which was the difference between the maximum and minimum MIs among all DMSs, were similar to that of average of healthy controls (online supplementary table S3). In addition, the SD of the MI difference at each DMS between the patients and healthy controls were small (online supplementary table S3). These data indicated that LOM occurred evenly at all DMSs within the IGF2/H19 domain in these four patients.

On the other hand, SRS-s09 showed an uneven methylation profile. CTS1-3 within the centromeric block of ICR1 were hypomethylated; however, CTS4-6 within the telomeric block showed normal methylation (figure $1 \mathrm{~B}$ right, online supplementary table S3). Bisulfite sequencing revealed normal differential methylation of CTS5, where the two parental alleles could be distinguished using the single-nucleotide variant rs2735972 (online supplementary figure S3). Furthermore, IGF2-DMR0 and IGF2-DMR2 (located far upstream of ICR1) also showed LOM, whereas CTS7 and the H19-promoter (located downstream of ICR1) showed normal methylation (figure 1B right, online supplementary table S3). Long-range PCR encompassing the entire ICR1 did not show any deletions or insertions (online supplementary figure S4B). Sequencing analysis, including seven CTSs; three OCT4/SOX2 sites; and 12 ZFP57 sites, detected known SNVs, but not a pathogenic variant (online supplementary table S4). MLPA showed two parental copies of $11 \mathrm{p} 15$ (online supplementary figure S4A). Moreover, all minor causative alterations for SRS, including pathogenic variants of the IGF2 and CDKN1C genes (data not shown), aberrant methylation of MEG3 at $14 \mathrm{q} 32.2$ and maternal UPDs of chromosomes 14,16 and 20, were not found (online supplementary figure S4C and S5). Sequencing analysis of ZFP57 also did not detect any variation (data not shown). Furthermore, the karyotype of this patient was also normal (data not shown). Taken together, the LOM of the ICR1 centromeric block was strongly suggested as a causative alteration in this patient.

\section{DISCUSSION}

In this study, we analysed 10 DMSs within the IGF2/H19 domain in five SRS patients with isolated ICR1-LOM. We found that four patients showed an evenly reduced DNA methylation at all DMSs. However, one patient (SRS-s09) showed a peculiar pattern of LOM, which was LOM at DMSs within the centromeric region of the IGF2/H19 domain and maintained methylation at DMSs within the telomeric region. This peculiar LOM suggested several important points in terms of an imprinting mechanism and molecular diagnosis. First, DNA methylation of the two repeat blocks within ICR1 may be independently regulated. CTCF, OCT4 and SOX2, which bind to their own motifs in ICR1, are important trans-factors for unmethylated maternal ICR1. ${ }^{8}$ ZFP57 and ZBTB33 are also important for the maintenance of the methylated paternal ICR1. ${ }^{5}$ Binding of these factors is thought to regulate the methylation of the entire ICR1 region. We did not find any pathogenic variants in these binding motifs or any putative variants associated with LOM of paternal ICR1. We also did not find any variation of ZFP57. This suggests the possible existence of other regulatory element(s) outside of ICR1, probably bound by a known or unknown trans-factor, which independently regulate the methylation of the centromeric and telomeric ICR1 blocks. In addition, the difference in methylation status between the two blocks within ICR1 also suggests that the centromeric block may be more susceptible to LOM than the telomeric block. 
Secondly, DNA methylation of IGF2-DMR0 and IGF2-DMR2 may be under the control of the centromeric block of ICR1, but not the telomeric block. Additionally, DNA methylation of the H19-promoter may be under the control of the telomeric block, but not the centromeric block. In general, somatic DMRs (eg, IGF2-DMRs) are established after fertilisation in response to nearby gametic DMRs (eg, ICR1). ${ }^{2}{ }^{17}$ In mice, ICR1 is needed on the maternal allele to protect the Igf2-DMRs from methylation after fertilisation through long-range chromatin interactions, although it is unknown whether each half of the mouse ICR1 has different functions. ${ }^{18}$ The results observed for SRSs09 suggest that half of the ICR is sufficient to affect the establishment of somatic DMRs, including the IGF2-DMRs and the H19-promoter.

Thirdly, IGF2 expression may be repressed by LOM of the centromeric block, and this reduction may be sufficient for the clinical features of SRS to develop. Of note, we were unable to examine IGF2 expression because of the unavailability of RNAs from IGF2-expressing tissues. We presume that LOM of the centromeric block induced the insulator activity of ICR1 and the change of the higher-order chromatin conformation of the IGF2/ $H 19$ domain, resulting in IGF2 repression in this patient. ${ }^{8} 19$ Since the methylation of the H19-promoter was normal, H19 expression is expected to be maintained at a normal level.

Lastly, the methylation statuses of both the centromeric and telomeric blocks must be analysed to reach a correct molecular diagnosis. Although peculiar LOM patterns may be rare, CTSs in both the centromeric and telomeric blocks must be simultaneously analysed to avoid a molecular misdiagnosis.

The peculiar LOM of the patient with SRS reveals unknown mechanisms of ICR1 and emphasises the need for precise molecular methods to appropriately determine the methylation status of ICR1. The new mechanisms of ICR1 should be elucidated to understand the imprinting mechanism of the IGF2/H19 domain.

Acknowledgements We thank the Analytical Research Center for Experimental Sciences, Saga University, for the experimental support.

Contributors $\mathrm{KH}$ and $\mathrm{HS}$ designed the study, interpreted the results and wrote the manuscript; HW, HT and TT provided the patient samples; YT, SH, HY and KH performed almost all experiments. All coauthors participated in the review of the manuscript and have approved the final version.

Funding This study was supported in part by: grants from the Grant-in-Aid for Scientific Research (C) program of the Japan Society for the Promotion of Science (16K09970 to KH; 17 K08687 to HS); grants for Practical Research Projects for Rare/ Intractable Diseases from the Japan Agency for Medical Research and Development (AMED) (17ek0109280h0001, 17ek0109234h0001 and 17ek0109205h0001 to HS); and a grant for Research on Intractable Diseases from the Ministry of Health, Labor, and Welfare (H29-nanchitou(nan)-ippan-025 to HS).

Competing interests None declared.

Patient consent for publication Not required.

Ethics approval This study was approved by the Ethics Committee for Human Genome and Gene Analyses of the Faculty of Medicine, Saga University.

Provenance and peer review Not commissioned; externally peer reviewed.

Open access This is an open access article distributed in accordance with the Creative Commons Attribution Non Commercial (CC BY-NC 4.0) license, which permits others to distribute, remix, adapt, build upon this work non-commercially, and license their derivative works on different terms, provided the original work is properly cited, appropriate credit is given, any changes made indicated, and the use is non-commercial. See: http://creativecommons.org/licenses/by-nc/4.0/.

ORCID iD

Hidenobu Soejima http://orcid.org/0000-0001-5076-6563

\section{REFERENCES}

1 Wakeling EL, Brioude F, Lokulo-Sodipe O, O'Connell SM, Salem J, Bliek J, Canton APM, Chrzanowska KH, Davies JH, Dias RP, Dubern B, Elbracht M, Giabicani E, Grimberg A, Grønskov K, Hokken-Koelega ACS, Jorge AA, Kagami M, Linglart A, Maghnie M, Mohnike K, Monk D, Moore GE, Murray PG, Ogata T, Petit IO, Russo S, Said E, Toumba M, Tümer Z, Binder G, Eggermann T, Harbison MD, Temple IK, Mackay DJG, Netchine I. Diagnosis and management of Silver-Russell syndrome: first international consensus statement. Nat Rev Endocrinol 2017;13:105-24.

2 Soejima $H$, Higashimoto K. Epigenetic and genetic alterations of the imprinting disorder Beckwith-Wiedemann syndrome and related disorders. J Hum Genet 2013;58:402-9.

3 Monk D, Mackay DJG, Eggermann T, Maher ER, Riccio A. Genomic imprinting disorders: lessons on how genome, epigenome and environment interact. Nat Rev Genet 2019:20:235-48.

4 Abi Habib W, Azzi S, Brioude F, Steunou V, Thibaud N, Das Neves C, Le Jule M, Chantot-Bastaraud S, Keren B, Lyonnet S, Michot C, Rossi M, Pasquier L, Gicquel C, Rossignol S, Le Bouc Y, Netchine I. Extensive investigation of the Igf2/H19 imprinting control region reveals novel OCT4/SOX2 binding site defects associated with specific methylation patterns in Beckwith-Wiedemann syndrome. Hum Mol Genet 2014:23:5763-73.

5 Bohne F, Langer D, Martiné U, Eider CS, Cencic R, Begemann M, Elbracht M, Bülow L, Eggermann T, Zechner U, Pelletier J, Zabel BU, Enklaar T, Prawitt D. Kaiso mediates human ICR 1 methylation maintenance and $\mathrm{H} 19$ transcriptional fine regulation. Clin Epigenetics 2016;8:47.

6 Sparago A, Cerrato F, Riccio A. Is ZFP57 binding to H19/IGF2:IG-DMR affected in Silver-Russell syndrome? Clin Epigenetics 2018;10:23.

7 Kraft F, Wesseler K, Begemann M, Kurth I, Elbracht M, Eggermann T. Novel familial distal imprinting centre $1(11 \mathrm{p} 15.5)$ deletion provides further insights in imprinting regulation. Clin Epigenetics 2019;11:30.

8 Shmela ME, Gicquel CF. Human diseases versus mouse models: insights into the regulation of genomic imprinting at the human $11 \mathrm{p} 15 /$ mouse distal chromosome 7 region. J Med Genet 2013;50:11-20.

9 Demars J, Shmela ME, Rossignol S, Okabe J, Netchine I, Azzi S, Cabrol S, Le Caignec C, David A, Le Bouc Y, El-Osta A, Gicquel C. Analysis of the Igf2/H19 imprinting control region uncovers new genetic defects, including mutations of OCT-binding sequences, in patients with $11 \mathrm{p} 15$ fetal growth disorders. Hum Mol Genet 2010;19:803-14.

10 Higashimoto K, Jozaki K, Kosho T, Matsubara K, Fuke T, Yamada D, Yatsuki H, Maeda T, Ohtsuka Y, Nishioka K, Joh K, Koseki H, Ogata T, Soejima H. A novel de novo point mutation of the OCT-binding site in the IGF2/H19-imprinting control region in a Beckwith-Wiedemann syndrome patient. Clin Genet 2014;86:539-44.

11 Sun F, Higashimoto K, Awaji A, Ohishi K, Nishizaki N, Tanoue Y, Aoki S, Watanabe H, Yatsuki $H$, Soejima $H$. The extent of DNA methylation anticipation due to a genetic defect in ICR1 in Beckwith-Wiedemann syndrome. J Hum Genet 2019;64:937-43.

12 Li X, Ito M, Zhou F, Youngson N, Zuo X, Leder P, Ferguson-Smith AC. A maternalzygotic effect gene, Zfp57, maintains both maternal and paternal imprints. Dev Cell 2008:15:547-57

13 Quenneville S, Verde G, Corsinotti A, Kapopoulou A, Jakobsson J, Offner S, Baglivo I, Pedone PV, Grimaldi G, Riccio A, Trono D. In embryonic stem cells, ZFP57/KAP1 recognize a methylated hexanucleotide to affect chromatin and DNA methylation of imprinting control regions. Mol Cell 2011:44:361-72.

14 Azzi S, Steunou V, Tost J, Rossignol S, Thibaud N, Das Neves C, Le Jule M, Habib WA, Blaise A, Koudou Y, Busato F, Le Bouc Y, Netchine I. Exhaustive methylation analysis revealed uneven profiles of methylation at IGF2/ICR 1/H19 11p15 loci in Russell silver syndrome. J Med Genet 2015;52:53-60.

15 Hidaka H, Higashimoto K, Aoki S, Mishima H, Hayashida C, Maeda T, Koga Y, Yatsuki H, Joh K, Noshiro H, Iwakiri R, Kawaguchi A, Yoshiura K-I, Fujimoto K, Soejima H. Comprehensive methylation analysis of imprinting-associated differentially methylated regions in colorectal cancer. Clin Epigenetics 2018;10:150.

16 Yatsuki H, Higashimoto K, Jozaki K, Koide K, Okada J, Watanabe Y, Okamoto N, Tsuno Y, Yoshida Y, Ueda K, Shimizu K, Ohashi H, Mukai T, Soejima H. Novel mutations of CDKN1C in Japanese patients with Beckwith-Wiedemann syndrome. Genes Genomics 2013;35:141-7.

17 Ferguson-Smith AC. Genomic imprinting: the emergence of an epigenetic paradigm. Nat Rev Genet 2011;12:565-75.

18 Lopes S, Lewis A, Hajkova P, Dean W, Oswald J, Forné T, Murrell A, Constância M, Bartolomei M, Walter J, Reik W. Epigenetic modifications in an imprinting cluster are controlled by a hierarchy of DMRs suggesting long-range chromatin interactions. Hum Mol Genet 2003;12:295-305.

19 Nativio R, Sparago A, Ito Y, Weksberg R, Riccio A, Murrell A. Disruption of genomic neighbourhood at the imprinted Igf2-H19 locus in Beckwith-Wiedemann syndrome and Silver-Russell syndrome. Hum Mol Genet 2011;20:1363-74. 